\title{
Higher baseline levels of CSF inflammation increase risk of incident mild cognitive impairment and Alzheimer's disease dementia
}

\section{Joey Annette Contreras}

University of Southern California Keck School of Medicine https://orcid.org/0000-0002-1778-3641

\section{Vahan Aslanyan}

USC Keck School of Medicine: University of Southern California Keck School of Medicine

\section{Daniel S Albrecht}

USC Keck School of Medicine: University of Southern California Keck School of Medicine

\section{Wendy J. Mack}

USC Keck School of Medicine: University of Southern California Keck School of Medicine

Judy Pa ( $\square$ judypa@usc.edu )

University of Southern California

\section{Research}

Keywords: Alzheimer's Disease, Conversion, Inflammation, CSF, ADNI

Posted Date: September 29th, 2021

DOI: https://doi.org/10.21203/rs.3.rs-917413/v1

License: (c) (i) This work is licensed under a Creative Commons Attribution 4.0 International License. Read Full License 


\section{Abstract \\ Background}

Few studies have investigated how inflammation early in the disease course may affect AD progression over time despite converging evidence that elevated levels of inflammation are associated with $A D$ in cross-sectional studies.

\section{Methods}

Two-hundred ninety-two research participants with CSF biomarkers from the Alzheimer's Disease Neuroimaging Initiative (ADNI) were included in this study. Cox proportional hazards models were used to investigate whether baseline levels of inflammatory CSF markers were associated with incident mild cognitive impairment or $A D$ and time to conversion. Potential moderating effects of sex and APOE4 were also examined.

\section{Results}

Elevated levels of pro-inflammatory markers TNF-a, IL-9, and IL-12p40 at baseline were associated with higher rates of conversion to $\mathrm{MCl} / \mathrm{AD}$. Interactions with sex and $A P O E 4$ were observed, such that women with elevated TNF- $a$ and all APOE4 carriers with elevated IL-9 levels had shorter times to conversion. Additionally, TNF-a mediated the relationship between elevated IL-12p40 and IL-9.

\section{Conclusion}

Elevated inflammation markers are associated with incident $\mathrm{MCl}$ or $\mathrm{AD}$, and the factors of sex and APOE4 status modify the time to conversion.

\section{Background}

Alzheimer's disease (AD) is increasingly recognized as a complex disease with heterogenous pathologies that affects populations and individuals differently. Numerous studies have demonstrated the importance of CSF biomarkers A 342 , phosphorylated-tau (p-tau), and total-tau (t-tau) as predictors of conversion to mild cognitive impairment $(\mathrm{MCl})$ or $A D$. However, relatively little is known about the role of inflammation in conversion, and even less about the interactions between inflammation and sex or APOE4 status.

A growing body of evidence indicates that neuroinflammatory responses exacerbate AD progression (14) and that inflammatory markers colocalize with $A D$ pathology postmortem in $A D$ clinical cohorts (5-9). Post-mortem studies of the AD brain indicate overexpression and/or activation of NF-KB, particularly in 
regions with high levels of $A \beta$ plaques and neurofibrillary tangles (NFT) (10). NF-KB is a key

transcriptional regulator of inflammation and, more recently, has also been found to have roles in learning and memory via neuronal signaling (11-12). NF-KB has binding sites in the promoter region of the genes involved in amyloidogenesis and inflammation (10) and as such, inflammatory markers associated with the NF-KB pathway are of particular interest and relevance to AD.

The cytokine tumor necrosis factor a (TNF-a), implicated in many AD studies, is among the most wellstudied and well-known activators of NF-KB in both neuronal and non-neuronal cells (13-14) and plays an important role in regulating long-term potentiation (LTP) (11). In addition to TNF-a, IL-9 is also a part of the NF-KB inflammatory pathway and has been associated with AD risk. Interleukin 9 (IL-9) is an understudied pro-inflammatory cytokine known for promoting cell proliferation and prevention of apoptosis and whose expression is regulated by NF-KB (15). Collectively, this pathway may play a central role in inflammation associated with $A D$ risk.

In this longitudinal study, we examined whether the baseline level of each CSF inflammatory marker was associated with incident $\mathrm{MCl}$ or $\mathrm{AD}$, and whether these markers interact to participate in the same inflammatory pathway. We hypothesized that neuroinflammatory markers (TNF- $a$, IL-9), along with the classic CSF biomarkers of AD (Aß42, t-tau, and p-tau), would predict clinical conversion to MCI/AD. Lastly, accompanying the current literature on sex and APOE4 differences in AD (16), we investigated the role of these two risk factors on inflammation and conversion in an exploratory analysis.

\section{Methods}

\section{Subjects}

Data used in the preparation of this manuscript were obtained from the Alzheimer's Disease Neuroimaging Initiative (ADNI) database (adni.loni.usc.edu). The ADNI was launched in 2003 as a publicprivate partnership, led by Principal Investigator Michael W. Weiner, MD. The primary goal of ADNI has been to test whether serial magnetic resonance imaging (MRI), positron emission tomography (PET), other biological markers, and clinical and neuropsychological assessment can be combined to measure the progression of mild cognitive impairment $(\mathrm{MCl})$ and early Alzheimer's disease (AD). ADNI is a convenience sample predominantly comprised of highly educated, non-Hispanic whites.

Participants were selected by availability of CSF data at baseline, and the availability of at least one additional time point for diagnosis as of January 2021. Additional participant demographics included APOE status, age, sex, and diagnosis at time of CSF collection ( $n=295 ; 177$ females). The combined sample excluded participants with AD diagnosis at the baseline visit. Participants who converted to $\mathrm{MCl}$ or $A D$ at the second time point were categorized as converters. Participants without diagnostic change throughout the observation period were categorized as non-converters. The frequency of follow-up visits consisted of a baseline visit, 3-month, 6-month, and annual visits. Local institutional review boards 
approved the ADNI study across the United States and Canada, and each subject provided written informed consent.

\section{APOE4 carrier status}

The details of $A P O E$ genotyping have been previously published (17). Participants with at least

one copy of the E4 allele were considered APOE4 carriers which consisted of primarily

heterozygous APOE3/4 genotypes (78\% APOE3/4; 19\% APOE4/4; 0.03\% APOE2/4).

\section{CSF markers}

According to the ADNI protocol, the levels of CSF inflammatory markers of interest (TNF-a, IL-9, IL-12p40) were measured examined by William Hu and J. Christina Howell, Department of Neurology, Emory University. All markers were measured in duplicate using commercially available multiplex immunoassays (Millipore Sigma, Burlington, MA, United States) (18). Values are given in pg/ml. Primary analyses examined TNF- $a$ and IL-9 to test the hypothesis that higher levels of inflammatory markers associated with transcription factor NF-kB would be associated with conversion. Exploratory analyses examined the CSF biomarker IL-12p40.

CSF AD biomarkers A 442 , t-tau and p-tau proteins were analyzed with the multiplex xMAP Luminex platform and Innogenetics/Fujirebio AlzBio3 immunoassay reagents, details of which have been described previously (19).

\section{Statistical Analyses}

Analyses were conducted using SPSS Statistics 27 (IBM Corp. Released 2020. IBM SPSS Statistics for Windows, Version 27.0. Armonk, NY: IBM Corp.) and R (R Core Team (2020). R: A language and environment for statistical computing. R Foundation for Statistical Computing, Vienna, Austria. URL: https://www.R-project.org/), and PASS 14 (PASS 14 Power Analysis and Sample Size Software (2015). NCSS, LLC. Kaysville, Utah, USA, ncss.com/software/pass.).

Baseline differences in CSF inflammatory marker levels between converters and non-converters were assessed by Wilcoxon rank sum. An association was considered significant if the two-sided $p$ value corresponding to the test statistic was less than 0.05 . Cox proportional hazards models were used to investigate whether the baseline levels for each CSF inflammatory marker and pathology markers (Aß42, p-tau, and t-tau) were associated with hazard rate of conversion. Lower CSF A $\beta$-42 values is an indicator of greater presence of amyloid-beta in the brain, which is contrary to the other CSF markers in which higher levels indicate greater $A D$ risk. Therefore, for $A \beta 42$ values only, the inverse $H R$ is reported $(1 / H R)$ to align lower $A \beta 42$ values with increased $A D$, which places all CSF markers on the same scale of $H R>1.0$ equates to elevated risk of conversion. CSF inflammatory markers were scaled to have a median of 0 and interquartile range (IQR) of 1 for interpretation purposes; effect estimates, expressed as hazard ratios (HRs) are therefore interpreted per IQR. Baseline age, sex, and APOE4 status were included in the analyses 
as covariates. Schoenfeld residuals were visually inspected to test for violation of the proportional hazards' assumption. Plots of Martingale residuals against continuous covariates were used to detect nonlinearity. Potential moderating effects of sex and APOE4 were tested by assessing CSF marker*sex and CSF marker* $A P O E 4$ interactions using females and $A P O E 4$ non-carriers as the corresponding reference groups. Further stratification and repeated analyses were conducted if the $\mathrm{p}$-value corresponding to an interaction term was below 0.15 (20).

Power analyses for Cox regression were carried out using PASS 14 software. The assumption for validity of proportional hazards model was tested before carrying out the analysis. Standard deviations of the variables of interest were calculated for power analyses. The sample size was adjusted based on the variability in the continuous variables of interest explained by the additional covariates in the Cox regression. The sample size was adjusted for the anticipated event rate. We assumed $80 \%$ power and a 2sided alpha of 0.05 and calculated the minimum detectable regression coefficient (log HR) for the analyses at 0.2410 . This corresponds to HR of 1.27 .

A mediation analysis was carried out using the PROCESS macro (21) version 3.5.2 for SPSS to test the significance of associations between CSF inflammatory markers in NF-KB pathway. TNF-a was treated as a potential mediator in IL-12p40-IL-9 relationship $(x=I L-12 p 40, y=I L-9)$ based on a priori knowledge $(22,23)$ using age and sex as covariates.

\section{Results}

\section{Demographic characteristics of the cohort}

292 participants (152 Converters, 140 Non-converters) were included in this study excluding 3 participants due to outlying CSF values. Of the total sample, 118 participants (40\%) were female, 123 (42\%) were APOE4 carriers, and 108 (37\%) were cognitively unimpaired. CSF markers A $A 42, \mathrm{t}$-tau, and ptau differed between groups with increased levels of pathology in converters. Between converters and non-converters, there were no significant differences in age or CSF levels of TNF-a, IL-9 or IL-12-p40 at baseline. All variables of interest are summarized in Table 1. 
Table 1

Baseline demographic and biomarker values of research participants. Effect size and p-value correspond to group differences between participants who go on to convert (converters) and those that remain at the same impairment stage (non-converters). Medians and interquartile ranges were reported for continuous variables and counts, and corresponding percentages were reported for categorical variables. Continuous variable medians were compared using Wilcoxon rank sum test and effect size was calculated according

to Cohen's D. Categorical variable counts were compared using $\chi^{2}$ test and the respective size was calculated according to Cramer's V.

\begin{tabular}{|c|c|c|c|c|c|}
\hline Baseline variable & All $(n=292)$ & $\begin{array}{l}\text { Converter }(n= \\
152)\end{array}$ & $\begin{array}{l}\text { Non-converter }(n= \\
140)\end{array}$ & $\begin{array}{l}\text { Effect } \\
\text { Size }\end{array}$ & $\mathrm{p}_{\text {value }}$ \\
\hline $\operatorname{Sex}(M / F)$ & $174 / 118$ & $97 / 55$ & $77 / 63$ & 0.08 & 0.16 \\
\hline $\begin{array}{l}\text { APOE4 (Carrier/Non- } \\
\text { Carrier) }\end{array}$ & $123 / 169$ & $76 / 76$ & $47 / 93$ & 0.16 & $\dot{0} 01$ \\
\hline Age & $74.85(6.78)$ & $74.50(7.07)$ & $75.23(6.46)$ & 0.10 & 0.39 \\
\hline TNF- $a$ & $1.74(0.52)$ & $1.80(0.53)$ & $1.68(0.51)$ & 0.23 & 0.05 \\
\hline IL-9 $(n=212)$ & $3.44(1.55)$ & $3.58(1.69)$ & $3.30(1.38)$ & 0.18 & 0.19 \\
\hline IL-12 (P40) ( $=212)$ & $1.31(1.76)$ & $1.52(2.21)$ & $1.08(1.06)$ & 0.25 & 0.07 \\
\hline p-Tau $(\mathrm{N}=288)$ & $27.70(13.10)$ & $31.42(13.72)$ & $23.76(11.11)$ & 0.61 & $\hat{0.01}$ \\
\hline Tau $(\mathrm{N}=288)$ & $\begin{array}{l}287.48 \\
(114.95)\end{array}$ & $\begin{array}{l}318.49 \\
(119.16)\end{array}$ & $252.82(99.58)$ & 0.60 & $\dot{0} 01$ \\
\hline$A \beta-42$ & $\begin{array}{l}1018.82 \\
(581.56)\end{array}$ & $\begin{array}{l}870.28 \\
(578.58)\end{array}$ & $1180.10(545.27)$ & 0.55 & $\hat{0} .01$ \\
\hline
\end{tabular}

The median follow-up period was 2.13 years $(I Q R=3.21)$ after the baseline visit for converters and 3.22 years (4.44) for non-converters. For converters, median time to conversion to AD was 1.99 years (IQR = 2.97 years), whereas conversion to $\mathrm{MCl}$ was 5.32 years (IQR $=3.67$ years). $14(4.79 \%) \mathrm{CN}$ participants converted to AD, 22 (7.53\%) CN participants converted to $\mathrm{MCl}$, and $114(39.04 \%) \mathrm{MCl}$ participants converted to $A D$.

\section{CSF Biomarkers at baseline predict conversion to $\mathrm{MCl} / \mathrm{AD}$ :}

\section{Amyloid Beta-42}

Baseline $A \beta 42$ was significantly associated with increased rate of conversion to $\mathrm{MCl} / \mathrm{AD}$ after adjusting for baseline age, sex, and APOE4 carrier status (HR $=2.17$ per 1 IQR, $95 \% \mathrm{Cl}:(1.59,3.03), \mathrm{p}<0.001$, Fig. 1$)$. As expected, there was an $A \beta 42{ }^{\star} A P O E 4$ interaction on conversion ( $\mathrm{HR}=1.96$ per $1 \mathrm{IQR}, \mathrm{Cl}:(0.92,4.17), \mathrm{p}=$ 0.08). Lower $A \beta 42$ was significantly associated with increased rate of conversion, in both carriers $(H R=$ 3.33 per 1 IQR, $\mathrm{Cl}:(1.69,6.67), \mathrm{p}<0.001)$ and non-carriers (HR $=1.72$ per 1 IQR, $95 \% \mathrm{Cl}:(1.19,2.5), \mathrm{p}=$ $0.004)$. In a subgroup analysis of those who converted to $A D$, lower CSF $A \beta 42$ was significantly associated with increased rate of conversion to $A D(H R=3.23$ per 1 IQR, 95\% Cl: $(2.17,4.76), p<0.001)$. 
There was also a significant interaction with sex (HR=0.55 per 1 IQR, 95\% Cl: $(0.25,0.93), p=0.03)$, such that the rate of conversion among women was significantly different from men. $A \beta 42$ had higher rates of conversion in women ( $\mathrm{HR}=4.76$ per $1 \mathrm{IQR}, 95 \% \mathrm{Cl}$ : $(2.17,11.11), \mathrm{p}<0.001)$, which was also true among men $(\mathrm{HR}=2.63$ per $1 \mathrm{IQR}, 95 \% \mathrm{Cl}:(1.64,4.17), \mathrm{p}<0.001)$. There was no significant $\mathrm{A} \beta 42 \star A P O E 4$ interaction on conversion ( $\mathrm{HR}=0.66$ per $1 \mathrm{IQR}, 95 \% \mathrm{Cl}:(0.28,1.59), p=0.36)$. CSF $A \beta 42$ levels at baseline were not significantly associated with conversion to $\mathrm{MCl}(\mathrm{HR}=0.77$ per 1 IQR, $95 \% \mathrm{Cl}:(0.45,1.33), \mathrm{p}=$ 0.35).

\section{Phosphorylated-tau}

Higher baseline p-tau was significantly associated with increased rate of conversion to $\mathrm{MCl} / \mathrm{AD}(\mathrm{HR}=$ 1.64 per 1 IQR, 95\% Cl: $(1.35,1.98), p<0.001$, Fig. 1). No sex interactions were observed $(H R=0.9237$ per 1 IQR, 95\% Cl: $(0.65,1.32), \mathrm{p}=0.66)$. There was a significant $\mathrm{p}$-tau*APOE4 interaction on conversion $(\mathrm{HR}=$ 0.65 per IQR, Cl: $(0.44,0.97), p=0.04)$, such that higher $p$-tau levels were significantly associated with increased conversion among non-carriers ( $\mathrm{HR}=2.10$ per 1 IQR, 95\% Cl: $(1.54,2.87), \mathrm{p}<0.001)$, but not among APOE4 carriers $(\mathrm{HR}=1.28, \mathrm{Cl}:(0.99,1.66), \mathrm{p}=0.06)$ in APOE4-stratified models. There was a significant main effect of $p$-tau on the rate of conversion to AD (HR $=1.69$ per 1 IQR, 95\% Cl: $(1.38,2.07)$, $\mathrm{p}<0.001)$. There was a significant $\mathrm{p}$-tau*APOE4 interaction on conversion $(\mathrm{HR}=0.55,95 \% \mathrm{Cl}$ : $(0.36,0.85), p=0.01)$, such that $A P O E 4$ carriers had higher risk of conversion ( $\mathrm{HR}=2.43,95 \% \mathrm{Cl}:(1.72$, $3.43), \mathrm{p}<0.001)$ compared to APOE4 non-carriers $(\mathrm{HR}=1.31(1.00,1.71), \mathrm{p}=0.05)$. P-tau was not significantly associated with the rate of conversion to $\mathrm{MCl}(\mathrm{HR}=1.58,95 \% \mathrm{Cl}:(0.97,2.57), \mathrm{p}=0.07)$.

\section{Total-tau}

Higher baseline t-tau was significantly associated with increased rate of conversion to $\mathrm{MCl} / \mathrm{AD}(\mathrm{HR}=1.64$ per 1 IQR, 95\% Cl: $(1.35,1.99), p<0.001$, Fig. 1). No sex $(p=0.5)$ or APOE4 $(p=0.15)$ interactions with t-tau on conversion were observed. T-tau was significantly associated with a higher rate of conversion to $A D$ $(\mathrm{HR}=1.73$ per $1 \mathrm{IQR}, 95 \% \mathrm{Cl}:(1.4,2.14), \mathrm{p}<0.001)$. No t-tau*sex interaction on conversion to AD was observed. There was a significant t-tau*APOE4 interaction on conversion to AD (HR $=0.62$ per $1 \mathrm{IQR}, 95 \%$ $\mathrm{Cl}(0.40,0.96), \mathrm{p}=0.03)$, such that $A P O E 4$ carrier participants had higher hazard ratio for conversion (HR $=2.28$ per 1 IQR, 95\% Cl: $(1.63,3.18), p<0.001)$, compared to non-carriers $(\mathrm{HR}=1.34$ per 1 IQR, 95\% Cl: $(1.01,1.78), p=0.04)$. CSF t-tau levels at baseline were not significantly associated with conversion to $\mathrm{MCl}(\mathrm{HR}=1.72$ per $1 \mathrm{IQR}, 95 \% \mathrm{Cl}:(0.98,3.03), \mathrm{p}=0.06)$.

\section{Elevated CSF Inflammatory Markers at baseline predict conversion to $\mathrm{MCl} / \mathrm{AD}$ :}

\section{Tumor Necrosis Factor-a}

Baseline TNF-a level was significantly associated with rate of conversion to $M C l / A D(H R=1.24$ per 1 IQR, 95\% Cl:(1.01,1.52), $p=0.04$, Fig. 1, Fig. 2A). A significant TNF-a*sex interaction was observed $(\mathrm{HR}=0.81$ per 1 IQR change, $95 \% \mathrm{Cl}:(0.62,1.06), p=0.13)$, suggesting that the association between TNF- $a$ and 
conversion was higher among women ( $\mathrm{HR}=1.38,95 \% \mathrm{Cl}:(1.05,1.83), \mathrm{p}=0.02)$, but not among men $(\mathrm{HR}=$ 1.06 per 1 IQR, $95 \% \mathrm{Cl}:(0.99,1.127), p=0.06)$ in sex-stratified models. No TNF- $\alpha{ }^{\star} A P O E 4$ interactions on conversion were observed. There was a significant main effect of TNF-a on the rate of conversion to $A D$ $(\mathrm{HR}=1.33$ per IQR, 95\% Cl: $(1.04,1.71), p=0.02$, Fig. $2 \mathrm{C})$. A TNF- ${ }^{\star}$ sex interaction on conversion (HR = 0.69 per $1 \mathrm{IQR}, 95 \% \mathrm{Cl}:(0.42,0.86), \mathrm{p}=0.15)$ indicated that the association between TNF-a and conversion was higher among women $(\mathrm{HR}=1.66$ per $1 \mathrm{IQR}, 95 \% \mathrm{Cl}:(1.1,2.48), \mathrm{p}=0.01)$ than men $(\mathrm{HR}=1.08$ per 1 IQR, 95\% Cl: $(0.78,1.5), p=0.63)$. TNF-a was not significantly associated with rate of conversion to $\mathrm{MCl}$ $(\mathrm{HR}=1.36$ per IQR change, $95 \% \mathrm{Cl}:(0.87,2.11), \mathrm{p}=0.18, \mathrm{Fig} .2 \mathrm{~B})$. There were no significant interactions with $A P O E 4$ carrier status in the models.

\section{Interleukin-9}

Baseline IL-9 level was not significantly associated with the rate of conversion to $\mathrm{MCl} / \mathrm{AD}(\mathrm{HR}=1.07$ per 1 IQR, 95\% Cl: $(0.84,1.37), p=0.56$, Fig. $3 A)$ or with rate of conversion to $A D(H R=1.07$ per 1 IQR, 95\% Cl: $(0.81,1.39), p=0.65)$ (Fig. $3 C$ ). No significant interaction with sex or APOE4 and IL-9 on conversion was observed. Baseline IL-9 levels were significantly associated with a higher rate of conversion to $\mathrm{MCl}(\mathrm{HR}=$ 1.53 per IQR change, 95\% Cl: $(3.97,5935.36), p=0.01)$ (Fig. 1, 3B). A significant IL-9*APOE4 interaction $(\mathrm{HR}=0.001,95 \% \mathrm{Cl}:(<0.001,0.17), \mathrm{p}=0.01)$ on $\mathrm{CN}-\mathrm{MCl}$ conversion, indicating that IL-9 conversion was significant in APOE4 carriers ( $\mathrm{HR}=1.39$ per $1 \mathrm{IQR}, 95 \% \mathrm{Cl}$ : $(0.37,52713), \mathrm{p}=0.10)$, but not in non-carriers $(\mathrm{HR}=1.04$ per $1 \mathrm{IQR}, 95 \% \mathrm{Cl}:(0.5,2.23), \mathrm{p}=0.91)$ in APOE4-stratified models. There was no significant interaction with IL-9*sex on conversion to $\mathrm{MCl}$.

\section{Interleukin-12p40}

Baseline levels of IL-12p40 were significantly associated with a higher rate of conversion to MCI/AD (HR $=1.38$ per 1 IQR, 95\% Cl: $(1.20,1.59)$, p < 0.01, Figs. 1, 4A). No sex or APOE4 interactions with IL-12p40 on conversion were observed. IL-12p40 was significantly associated with conversion to $A D(H R=1.36$ per 1 IQR, 95\% Cl: (1.18,1.57), $p<0.01$, Fig. 4C). IL-12p40 was not significantly associated with conversion to $\mathrm{MCl}(\mathrm{HR}=1.42$ per 1 IQR, 95\% Cl: (0.72,2.815), $\mathrm{p}=0.31$, Fig. 4B).

\section{TNF- $a$ mediates the association between IL-12p40 and IL-9}

Based on a priori knowledge of the NF-KB relationship between the three inflammatory markers (IL-12p40, IL-9, and TNF-a), a bootstrapped mediation analysis was conducted to investigate whether TNF- $\mathrm{a}(\mathrm{M})$ was a significant mediator of IL-12p40 (X) and IL-9 (Y). Adjusting for age and sex, IL-12p40 was significantly associated with TNF-a $(\beta=0.07 \mathrm{per} 1 \mathrm{pg} / \mathrm{mL}, \mathrm{Cl}:(0.04,0.11), p<0.001)$ and with IL-9 $(\beta=$ 0.12 per $1 \mathrm{pg} / \mathrm{mL}$, Cl: $(0.00,0.24), \mathrm{p}=0.04)$. TNF-a was a significant mediator in IL-12p40-IL-9 relationship. The indirect effect (average mediated effect) was 0.11 (95\% Cl: $(0.05,0.18), p<0.001)$. Average direct effect was 0.01 (95\% Cl: $(-0.10,0.12), p=0.81)$. Total effect was 0.13 (95\% Cl: $(0.01,0.24)$, $\mathrm{p}=0.04)$. TNF-a mediated $88.64 \%$ of the relationship $(95 \% \mathrm{Cl}:(0.33,5.22), \mathrm{p}=0.04$, Fig. 5). 


\section{Discussion}

Chronic low-grade inflammation is a hallmark feature of $A D$, as evidenced by increased expression of proinflammatory cytokines in postmortem AD brains (24-26), yet outside of examining known CSF biomarkers, few studies have examined the contribution of elevated inflammatory levels on future incidence of clinical conversion. In the present study, we evaluated inflammatory markers at baseline to determine differences between those who progressed to $\mathrm{MCl}$ or $\mathrm{AD}$ and those that remained stable over time. Evaluating individuals before conversion to $A D$ retroactively evaluates early features of $A D$ risk and can provide an outlook on time to conversion. Therefore, the goal of this study was to address whether: i) inflammatory cytokines are involved in incident $\mathrm{MCl}$ or $\mathrm{AD}$ as compared to canonical CSF $A D$ biomarkers, ii) sex or $A P O E 4$ impact these relationships, and iii) there is a relationship between the inflammatory markers via mediation analysis.

As expected, CSF biomarkers $\mathrm{A} \beta 42$, $\mathrm{p}$-tau, and t-tau predicted conversion to $\mathrm{MCl}$ or $\mathrm{AD}$. This was especially true among APOE4 carriers for $A \beta 42$ and p-tau, which has been previously confirmed $(27,28)$. In line with the primary goal of the study, inflammatory CSF markers TNF-a, IL-9, and IL-12p40 were associated with conversion to $\mathrm{MCl} / \mathrm{AD}$ with sex and $A P O E 4$ interactions.

While we know that neural degeneration and the chronic presence of pathology can stimulate inflammation, evidence suggests that inflammation itself can both generate and increase pathology. For instance, modulatory proteins associated with innate immunity are directly connected to amyloid-beta production (29) indicating the importance of neuroimmune actions on AD. Therefore, it is possible that inflammatory markers may be important indicators of $\mathrm{MCl}$ and $\mathrm{AD}$ risk, possibly accelerating decline. Additionally, several interactions involving sex and APOE4 status among these inflammatory markers were observed, suggesting these genetic factors may influence the relationship between early inflammation and future AD risk, providing key insight for precision medicine approaches. Lastly, the association between these markers suggests a common tie to the NF-kB inflammatory pathway, which may be activated in AD.

CSF TNF-a, one of the inflammatory markers most consistently implicated in AD, was associated with conversion in individuals who were cognitively normal at baseline. Previous work has shown increased levels of TNF-a in both the brains and plasma of patients with clinical AD (30). This may be partially related to $A \beta$, which can directly stimulate microglial production of TNF- $\alpha$ through activation of the transcription factor NF-KB (31). In addition, early increases in TNF-a can increase A $\beta$ burden through the upregulation of $\beta$-secretase production and increase of $\gamma$-secretase activity $(32,33)$, demonstrating how TNF- $\alpha$ and $A \beta$ can perpetuate a vicious cycle of increasing pathology and chronic inflammation. In corroboration of a role for TNF- $a$ in AD, a strong association between elevated TNF-a levels and decreased functional connectivity in an $A D$ susceptible cohort has been observed at a whole-brain level (34). Aside from TNF-a as a consistently elevated proinflammatory marker in AD, its regulation of the NF$\mathrm{KB}$ inflammatory cascade is well characterized $(35,36)$. 
When decomposing the association between TNF-a levels in cognitively normal individuals and clinical converters, the effect was driven by conversion to $A D$, not conversion to $\mathrm{MCl}$, indicating that elevated levels of TNF-a resulted in accelerated onset of Alzheimer's dementia. Given that TNF-a both promotes and is regulated by NF-KB, it could be that TNF- $a$ accumulates at a faster rate than other inflammatory factors. In AD patients who experienced systemic inflammatory events (SIE), rapid cognitive decline was observed over 6 months creating a 2-fold decrease in cognitive function. Additionally, SIE AD patients also exhibited an association between elevated plasma TNF-a levels and a 10-fold greater rate of cognitive decline over a 6-month observation period (37).

Importantly, a sex*TNF-a interaction was observed in which women with higher TNF-a levels had shorter time to conversion to $\mathrm{MCl}$ or $\mathrm{AD}$. There is precedence for this result with recent evidence suggesting important sex differences in immune parameters. This includes brain responses to inflammatory mediators and markers of microglial disruption which may contribute to higher risk of AD among women (38). For instance, given an acute endotoxin challenge, females responded with higher levels of proinflammatory plasma cytokines like TNF- $a$, compared to males (39). This may be due to a putative link between levels of TNF- $a$ and sex hormones. A significant positive correlation between luteinizing hormone and TNF-a levels has been observed (albeit only in males) providing some evidence that sex hormones exert their influence on AD by modulating systemic TNF-a levels (40). There are also sex differences among function, morphology, and volume of microglia, with evidence suggesting that women later in life have higher numbers of microglia than men, creating an exaggerated immune response (41). Interestingly, our finding that females with higher TNF-a levels have a shorter time to conversion to $\mathrm{MCl} / \mathrm{AD}$ suggests that elevated inflammation is associated with $A D$ risk. It has been suggested that elevated inflammatory factors, including TNF- $a$, are associated with lower brain levels of amyloid-beta and tau in cognitively normal individuals (42), suggesting inflammation may be protective for a time before eventually exhausting this compensatory effect and converting to $A D$ at an accelerated rate. Studies have started showing prevention of cognitive loss with anti-TNF-a therapy in a mouse model of compromised cognition (43) lending additional evidence to an important role of TNF- $a$ in the initiation and amplification of the inflammatory cascade.

Baseline elevated IL-9 levels were associated with conversion to MCI or AD, but in contrast to TNF- $a$, the results were driven by conversion to $\mathrm{MCl}$ and not $\mathrm{AD}$. Additionally, no interaction with sex was observed. IL-9 is an understudied pro-inflammatory cytokine that promotes cell proliferation and whose expression is driven by NF-KB (44). Its pleiotropic effects have been implicated in both neurological (i.e., multiple sclerosis [45]) and systemic disorders (i.e., psoriasis [46]). Additionally, neurons are thought to possess the ability to induce T cells to release IL-9 [47]. Thus, IL-9 may represent a critical link in the crosstalk between neurons and perivascular T cells in the brain. Changes related to IL-9 have not been consistently reported in $\mathrm{AD}(48,49)$, but there is mouse $A P O E 4$ knock-in models showing greater IL-9 production than the wild-type $\varepsilon 3$ allele (50). We add to this evidence by reporting an interaction between APOE4 and IL-9 levels, such that APOE4 carriers with higher IL-9 levels at baseline had higher rates of conversion. 
Lastly, higher baseline levels of the proinflammatory cytokine IL-12p40 were also significantly associated with conversion to AD. IL-12, which has different subunits, including p40 as investigated here, is involved in the differentiation of naive T cells into Th1 cells [51] and plays an important role in the activities of natural killer (NK) cells and T lymphocytes, enhancing their activity. IL-12 also stimulates production of TNF-a from T and NK cells (52). Its role in AD remains unclear, but recent evidence indicates a role for IL12 in amyloid induced neurodegeneration (53). The p40 subunit of IL-12 is produced by microglia and was increased in transgenic mouse models demonstrating a significant correlation between cognitive performance and CSF IL-12p40 levels $(52,53)$. More support of its role in AD was shown by elevated CSF IL-12p40 levels in AD patients (54). Interestingly, the potential association of plasma IL-12p40 levels with the diagnosis of $\mathrm{MCl}$ or $\mathrm{AD}$ was also highlighted by a recent plasma multianalyte profiling study (55). Together, these findings imply that IL-12p40 is involved in AD progression.

To investigate interrelationships between cytokines showing significant associations with conversion to $\mathrm{MCl} / \mathrm{AD}$, mediation analyses using a priori knowledge of the markers and their role in the NF-KB pathway were conducted. As mentioned previously, IL-12p40 has been shown to stimulate the production of TNF-a (23), a canonical promotor of the NF-KB inflammatory pathway $(33,34)$, and evidence shows that IL-9 production is mediated by NF-KB (22). TNF- $\alpha$ was tested as a significant mediator between IL12-p40 and IL-9, and mediation analysis results suggest that TNF-a mediated $89 \%$ of the association between IL12p40 and IL-9. Based on the available literature, this study is the first showing that these cytokines may be involved in the same NF-KB inflammatory pathway, thereby further implicating the inflammatory cascade's importance in AD detection.

\section{Limitations}

Current limitations of this study include an inability to test specific demographic variables, including race, which we know play a significant role in inflammation and $A D$ and yet have not been prioritized in ADNI recruitment. The investigation of longitudinal cognitive data from this sample and other datasets could help determine if differences exist between converters and non-converters, and shed light on specific cognitive domains that may be more closely tied to inflammation. We also note that because this was interval censored data, we are not able to know the actual time of conversion and are restricted to examining data at discrete time points. Competing risks were not discussed (i.e., death occurring prior to follow-up). Additionally, because we only had baseline CSF inflammatory markers, we were restricted to a cross-sectional mediation analysis that limits our confidence in temporality of events, but as more data become available, longitudinal analyses of change in inflammatory markers can be evaluated. Lastly, the analyses include adjusted p-values to explore interactions, so the results should be carefully considered. Future studies aimed at replication of these findings in other independent cohorts, including those which are more nationally representative of the AD population, would help validate these results.

\section{Conclusions}


This work demonstrates the need to investigate inflammatory markers and their role in conversion to AD. Investigations focused on inflammation differences between key grouping, based on clinical diagnosis, sex, and $A P O E$ status may provide insight on key underlying processes relevant to future clinical conversion. As a testament to the importance of longitudinal analysis, no baseline differences in CSF inflammatory marker levels were observed between $\mathrm{CN}$ and $\mathrm{MCl}$ groups, or between APOE4 carriers and non-carriers in the current study. The strength of this study comes from the rich longitudinal dataset because changes occurring within individual participants may be more useful for characterizing disease progression than cross-sectional comparisons which are confounded/rendered less sensitive by large amounts of interindividual variation.

These inflammatory biomarkers could prove to be easily accessible targets to gauge the efficacy of therapeutic interventions and be crucial to point to new biological pathways that may play a role in dementia.

\section{Declarations}

\section{Ethics approval and consent to participate}

The study was approved by the institutional review boards of the participating institutions. All participants gave informed written consent. More details can be found online (https://adni.loni.usc.edu, Accessed: 17 September 2021).

\section{Consent for publication}

This manuscript meets the guidelines put forth by the Alzheimer's Disease Neuroimaging Initiative Data Sharing and Publication Policy.

Availability of data and materials

The data used in this publication are available through the ADNI website upon user approval: https://adni.loni.usc.edu

\section{Competing interests}

The authors declare that they have no competing interests.

\section{Funding}

J.A., V.A., and J.P. are supported by the National Institute on Aging (R01-AG054617). Data collection and sharing for this project was funded by the Alzheimer's Disease Neuroimaging Initiative (ADNI) (National Institutes of Health Grant U01 AG024904) and DOD ADNI (Department of Defense award number W81XWH-12-2-0012). ADNI is funded by the National Institute on Aging, the National Institute of Biomedical Imaging and Bioengineering, and through generous contributions from the following: AbbVie, Alzheimer's Association; Alzheimer's Drug Discovery Foundation; Araclon Biotech; BioClinica, Inc.; Biogen; 
Bristol-Myers Squibb Company; CereSpir, Inc.; Cogstate; Eisai Inc.; Elan Pharmaceuticals, Inc.; Eli Lilly and Company; Eurolmmun; F. Hoffmann-La Roche Ltd and its affiliated company Genentech, Inc.; Fujirebio; GE Healthcare; IXICO Ltd.; Janssen Alzheimer Immunotherapy Research \& Development, LLC.; Johnson \& Johnson Pharmaceutical Research \& Development LLC.; Lumosity; Lundbeck; Merck \& Co., Inc.; Meso Scale Diagnostics, LLC.; NeuroRx Research; Neurotrack Technologies; Novartis Pharmaceuticals Corporation; Pfizer Inc.; Piramal Imaging; Servier; Takeda Pharmaceutical Company; and Transition Therapeutics. The Canadian Institutes of Health Research is providing funds to support ADNI clinical sites in Canada. Private sector contributions are facilitated by the Foundation for the National Institutes of Health (www.fnih.org). The grantee organization is the Northern California Institute for Research and Education, and the study is coordinated by the Alzheimer's Therapeutic Research Institute at the University of Southern California. ADNI data are disseminated by the Laboratory for Neuro Imaging at the University of Southern California.

\section{$\underline{\text { Authors' contributions }}$}

Conceptualization: DSA; Implementation and computational methods: JAC \& VA; Paper writing: JCA; Paper review and supervision: JP. All authors read and approved the final manuscript.

\section{Acknowledgements}

*Data used in preparation of this article were obtained from the Alzheimer's Disease Neuroimaging Initiative (ADNI) database (adni.loni.usc.edu). As such, the investigators within the ADNI contributed to the design and implementation of $A D N I$ and/or provided data but did not participate in analysis or writing of this report. A complete listing of ADNI investigators can be found at: http://adni.loni.usc.edu/wpcontent/uploads/how_to_apply/ADNI_Acknowledgement_List.pdf

\section{Authors' Information}

Membership of the Alzheimer's Disease Neuroimaging Initiative is listed in the Acknowledgments.

Joey A Contreras, Vahan Aslanyan, Daniel S Albrecht, and Judy Pa are a part of the Mark and Mary Stevens Neuroimaging and Informatics Institute, Department of Neurology, University of Southern California, Los Angeles, CA, USA, Correspondence to Dr. Judy Pa

Wendy J Mack, Vahan Aslanyan are a part of the Department of Population and Public Health Sciences, Keck School of Medicine, University of Southern California, Los Angeles, CA, USA

\section{References}

1. Holtzman DM, Mandelkow E, Selkoe DJ. Alzheimer disease in 2020. Cold Spring Harb Perspect Med. 2012;2:a011585.

2. Perry VH, Cunningham $\mathrm{C}$, Holmes $\mathrm{C}$. Systemic infections and inflammation affect chronic neurodegeneration. Nat Rev Immunol. 2007;7:161-7. 
3. Bettcher BM, Kramer JH. Longitudinal inflammation, cognitive decline, and Alzheimer's disease: a mini-review. Clin Pharmacol Ther. 2014;96:464-9.

4. Wyss-Coray T, Rogers J. Inflammation in Alzheimer disease-a brief review of the basic science and clinical literature. Cold Spring Harb Perspect Med. 2012;2:a006346.

5. Janelidze S, Mattsson N, Stomrud E, et al. CSF biomarkers of neuroinflammation and cerebrovascular dysfunction in early Alzheimer disease. Neurology. 2018;28:e867-77.

6. Brosseron F, Traschütz A, Widmann $\mathrm{CN}$, et al. Characterization and clinical use of inflammatory cerebrospinal fluid protein markers in Alzheimer's disease. Alz Res Ther. 2018;10:25.

7. Whelan CD, Mattsson N, Nagle MW, et al. Multiplex proteomics identifies novel CSF and plasma biomarkers of early Alzheimer's disease. Acta Neuropathol Commun 2019;7:169. https://doi.org/10.1186/s40478-019-0795-2.

8. Suárez-Calvet M, Araque Caballero M, Kleinberger G, et al. Dominantly Inherited Alzheimer Network. Early changes in CSF STREM2 in dominantly inherited Alzheimer's disease occur after amyloid deposition and neuronal injury. Sci Transl Med. 2016;8:369ra178.

9. Puzzo D, Privitera L, Leznik E, Fa M, Staniszewski A, Palmeri A, et al. Picomolar amyloid-beta positively modulates synaptic plasticity and memory in hippocampus. J Neurosci. 2008;28(53):14537-45.

10. Ju Hwang C, Choi DY, Park MH, Hong JT. NF-KB as a Key Mediator of Brain Inflammation in Alzheimer's Disease. CNS Neurol Disord Drug Targets. 2019;18(1):3-10. doi: 10.2174/1871527316666170807130011. PMID: 28782486.

11. Jones SV, Kounatidis I. Nuclear Factor-Kappa B and Alzheimer Disease, Unifying Genetic and Environmental Risk Factors from Cell to Humans. Frontiers in immunology. 2017;8:1805. https://doi.org/10.3389/fimmu.2017.01805.

12. Snow WM, Albensi BC. Neuronal Gene Targets of NF-KB and Their Dysregulation in Alzheimer's Disease. Front Mol Neurosci. 2016;9:118. https://doi.org/10.3389/fnmol.2016.00118.

13. Hayden MS, Ghosh S. Regulation of NF-KB by TNF family cytokines. Seminars in immunology. 2014;26(3):253-66. https://doi.org/10.1016/j.smim.2014.05.004.

14. Kounatidis I, Ligoxygakis P. Drosophila as a model system to unravel the layers of innate immunity to infection. Open Biol (2012) 2(5):120075.10.1098/rsob.120075.

15. Early SB, Huyett P, Brown-Steinke K, Borish L, Steinke JW. Functional analysis of - 351 interleukin-9 promoter polymorphism reveals an activator controlled by NF-KB. Genes Immunity. 2009;10(4):3419.

16. Amélie Cartier M, Côté I, Lemieux L, Pérusse A, Tremblay C, Bouchard J-P, Després. Sex differences in inflammatory markers: what is the contribution of visceral adiposity? The American Journal of Clinical Nutrition Volume. May 2009;89(5):1307-14.

17. Nation DA, Sweeney MD, Montagne A, Sagare AP, D'Orazio LM, Pachicano M, Sepehrband F, Nelson AR, Buennagel DP, Harrington MG, Benzinger TL. Blood-brain barrier breakdown is an early biomarker of human cognitive dysfunction. Nature medicine. 2019 Feb;25(2):270-6. 
18. Hu WT, Holtzman DM, Fagan AM, Shaw LM, Perrin R, Arnold SE, Grossman M, Xiong C, CraigSchapiro R, Clark CM, Pickering E, Kuhn M, Chen Y, Van Deerlin VM, McCluskey L, Elman L, Karlawish J, Chen-Plotkin A, Hurtig HI, Siderowf A, Swenson F, Lee VM, Morris JC, Trojanowski JQ, Soares H (2012) Plasma multianalyte profiling in mild cognitive impairment and Alzheimer disease. Neurology 79, 897-905.

19. Shaw LM, Vanderstichele $H$, Knapik-Czajka M, Clark CM, Aisen PS, Petersen RC, Blennow K, Soares $H$, Simon A, Lewczuk P, Dean R, Siemers E, Potter W, Lee VM, Trojanowski JQ. Alzheimer's Disease Neuroimaging Initiative.

20. Ann N. 2009 Apr; 65(4):403-13.

21. Buckley JP, Doherty BT, Keil AP, Engel SM. Statistical Approaches for Estimating Sex-Specific Effects in Endocrine Disruptors Research. Environ Health Perspect. 2017;125:067013.

22. Hayes AF. Introduction to mediation, moderation, and conditional process analysis: A regressionbased approach. Guilford publications; 2017.

23. Goswami R, Kaplan MH. A brief history of IL-9. J Immunol. 2011 Mar 15;186(6):3283-8. doi: 10.4049/jimmunol.1003049. PMID: 21368237; PMCID: PMC3074408.

24. Zheng, Hua; Ban, Yi; Wei, Fang; Ma, Xiaojing (2016), Ma, Xiaojing (ed.), "Regulation of Interleukin-12 Production in Antigen-Presenting Cells", Regulation of Cytokine Gene Expression in Immunity and Diseases, Springer Netherlands, 941, pp. 117-138, doi:10.1007/978-94-024-0921-5_6, ISBN 978-94024-0919-2, PMID 27734411

25. Heppner FL, Ransohoff RM, Becher B. Immune attack: the role of inflammation in Alzheimer disease. Nat Rev Neurosci. 2015;16:358-72.

26. Kinney JW, Bemiller SM, Murtishaw AS, Leisgang AM, Salazar AM, Lamb BT. Inflammation as a central mechanism in Alzheimer's disease. Alzheimer's Dementia: Translational Research Clinical Interventions. 2018;4:575-90.

27. Swardfager W, Lanctôt K, Rothenburg L, Wong A, Cappell J, Herrmann N. A Meta- Analysis of Cytokines in Alzheimer's Disease. Biol Psychiat. 2010;68:930-41.

28. Fagan AM, Roe CM, Xiong C, Mintun MA, Morris JC, Holtzman DM. Cerebrospinal Fluid tau/ $\beta$ Amyloid42 Ratio as a Prediction of Cognitive Decline in Nondemented Older Adults. Arch Neurol. 2007;64(3):343-9. doi:10.1001/archneur.64.3.noc60123.

29. Blennow, K., Shaw, L.M., Stomrud, E. et al. Predicting clinical decline and conversion to Alzheimer's disease or dementia using novel Elecsys $A \beta(1-42)$, pTau and tTau CSF immunoassays. Sci Rep 9 , 19024 (2019). https://doi.org/10.1038/s41598-019-54204-z

30. Hur, JY., Frost, G.R., Wu, X. et al. The innate immunity protein IFITM3 modulates $\gamma$-secretase in Alzheimer's disease. Nature 586, 735-740 (2020). https://doi.org/10.1038/s41586-020-2681-2

31. Chang R, Yee K-L, Sumbria RK. Tumor necrosis factor a Inhibition for Alzheimer's Disease. J Cent Nervous Syst Dis. 2017;9117957351770927.

32. Combs CK, Karlo JC, Kao SC, Landreth GE. $\beta$-Amyloid stimulation of microglia and monocytes results in TNFalpha-dependent expression of inducible nitric oxide synthase and neuronal apoptosis. $J$ 
Neurosci. 2001;21:1179-88.

33. Liao Y-F, Wang B-J, Cheng H-T, Kuo L-H, Wolfe MS. Tumor Necrosis Factor-a, Interleukin-1 $\beta$, and Interferon-y Stimulate $\gamma$-Secretase-mediated Cleavage of Amyloid Precursor Protein through a JNKdependent MAPK Pathway. J Biol Chem. 2004;279:49523-32.

34. Yamamoto M, Kiyota T, Horiba M, Buescher JL, Walsh SM, Gendelman HE. Interferon-gamma and tumor necrosis factor-alpha regulate amyloid-beta plaque deposition and beta-secretase expression in Swedish mutant APP transgenic mice. Am J Pathol. 2007;170:680-92.

35. Contreras JA, Aslanyan V, Sweeney MD, Sanders L, Sagare AP, Zlokovic BV, Toga AW, Han SD, Morris JC, Fagan A, Massoumzadeh P, Benzinger TL, Pa J. Functional connectivity among brain regions affected in Alzheimer's disease is associated with CSF TNF-a in APOE4 carriers. Neurobiol Aging. 2020;86:112-22. https://doi.org/10.1016/j.neurobiolaging.2019.10.013.

36. Akiyama H, Barger S, Barnum S, Bradt B, Bauer J, Cole GM. Inflammation and Alzheimer's disease. Neurobiol Aging. 2000;21:383-421.

37. Fillit H, Ding WH, Buee L, Kalman J, Altstiel L, Lawlor B. Elevated circulating tumor necrosis factor levels in Alzheimer's disease. Neurosci Lett. 1991;129:318-20.

38. Holmes C., Cunningham C., Zotova E., Culliford D., Perry V.H. Proinflammatory cytokines, sickness behavior, and Alzheimer disease. Neurology. 2011;77:212-218.

39. Podcasy JL, Epperson CN. Considering sex and gender in Alzheimer disease and other dementias. Dialog Clin Neurosci. 2016;18(4):437-46. https://doi.org/10.31887/DCNS.2016.18.4/cepperson.

40. Engler H, Benson S, Wegner A, Spreitzer I, Schedlowski M, Elsenbruch S. (2016) Men and women differ in inflammatory and neuroendocrine responses to endotoxin but not in the severity of sickness symptoms. Brain, Behavior, and Immunity 52, 18-26.

41. Butchart JMA. MRCP*; Birch, Brian MD, FRCS ${ }^{\dagger}$; Bassily. Ramy BM*; Wolfe, Laura BSc ; Holmes, Clive PhD, MRCPsych* Male Sex Hormones and Systemic Inflammation in Alzheimer Disease, Alzheimer Disease \& Associated Disorders: April-June 2013 - Volume 27 - Issue 2 - p 153-156 doi: 10.1097/WAD.0b013e318258cd63.

42. Schwarz JM, Sholar PW, Bilbo SD. Sex differences in microglial colonization of the developing rat brain. Journal of neurochemistry. 2012;120:948-63.

43. Albrecht DS, Sagare A, Pachicano M, Sweeney MD, Toga A, Zlokovic B, Chui H, Joe E, Schneider L, Morris JC, Benzinger T, Pa J. Early neuroinflammation is associated with lower amyloid and tau levels in cognitively normal older adults. Brain Behav Immun. 2021 May;94:299-307. doi: 10.1016/j.bbi.2021.01.010. Epub 2021 Jan 22. PMID: 33486003.

44. Terrando N, Monaco C, Ma D, Foxwell BM, Feldmann M, Maze M. Tumor necrosis factor-alpha triggers a cytokine cascade yielding postoperative cognitive decline. Proc Natl Acad Sci USA. 2010;107(47):20518-22. https://doi.org/10.1073/pnas.1014557107.

45. Nowak EC, Weaver CT, Turner H, et al. IL-9 as a mediator of Th17-driven inflammatory disease. J Exp Med. 2009;206:1653-60. 
46. Li H, Nourbakhsh B, Ciric B, et al. Neutralization of IL-9 ameliorates experimental autoimmune encephalomyelitis by decreasing the effector T cell population. J Immunol. 2010;185:4095-100.

47. Schlapbach C, Gehad A, Yang C, et al. Human TH9 cells are skin-tropic and have autocrine and paracrine proinflammatory capacity. Sci TransI Med. 2014;6:219ra8.

48. Liu Y, Teige I, Birnir B, Issazadeh-Navikas S. Neuron-mediated generation of regulatory T cells from encephalitogenic T cells suppresses EAE. Nat Med. 2006;12:518-25.

49. Saresella M, Calabrese E, Marventano I, et al. Increased activity of Th-17 and Th-9 lymphocytes and a skewing of the post-thymic differentiation pathway are seen in Alzheimer's disease. Brain Behav Immun. 2011;25:539-47.

50. Stertz L, Contreras-Shannon V, Monroy-Jaramillo N, et al. BACE1-deficient mice exhibit alterations in immune system pathways. Mol Neurobiol. 2018;55:709-17.

51. Mace BE, Wang H, Lynch JR, et al. Apolipoprotein E modifies the CNS response to injury via a histamine-mediated pathway. Neurol Res. 2007;29:243-50.

52. Hsieh CS, Macatonia SE, Tripp CS, Wolf SF, O'Garra A, Murphy KM (April 1993). "Development of TH1 CD $4+$ T cells through IL-12 produced by Listeria-induced macrophages". Science. 260 (5107): 5479. doi:10.1126/science.8097338. PMID 8097338.

53. Pedrini S, Gupta VB, Hone E, et al. A blood-based biomarker panel indicates IL-10 and IL-12/23p40 are jointly associated as predictors of $\beta$-amyloid load in an AD cohort. Sci Rep. 2017;7:14057. https://doi.org/10.1038/s41598-017-14020-9.

54. Vom Berg J, Prokop S, Miller KR, Obst J, Kalin RE, Lopategui-Cabezas I, Wegner A, Mair F, Schipke CG, Peters O, Winter Y, Becher B, Heppner FL (2012) Inhibition of IL-12/IL-23 signaling reduces Alzheimer's disease-like pathology and cognitive decline. Nat Med 18, 1812-1819.

55. Tan MS, Yu JT, Jiang T, Zhu XC, Guan HS, Tan L. IL12/23 p40 inhibition ameliorates Alzheimer's disease-associated neuropathology and spatial memory in SAMP8 mice. J Alzheimers Dis. 2014;38(3):633 - 46. doi: 10.3233/JAD-131148. PMID: 24047617.

56. Hu WT, Holtzman DM, Fagan AM, Shaw LM, Perrin R, Arnold SE, Grossman M, Xiong C, CraigSchapiro R, Clark CM, Pickering E, Kuhn M, Chen Y, Van Deerlin VM, McCluskey L, Elman L, Karlawish J, Chen-Plotkin A, Hurtig HI, Siderowf A, Swenson F, Lee VM, Morris JC, Trojanowski JQ, Soares H. Plasma multianalyte profiling in mild cognitive impairment and Alzheimer disease. Neurology. 2012;79:897-905.

\section{Figures}




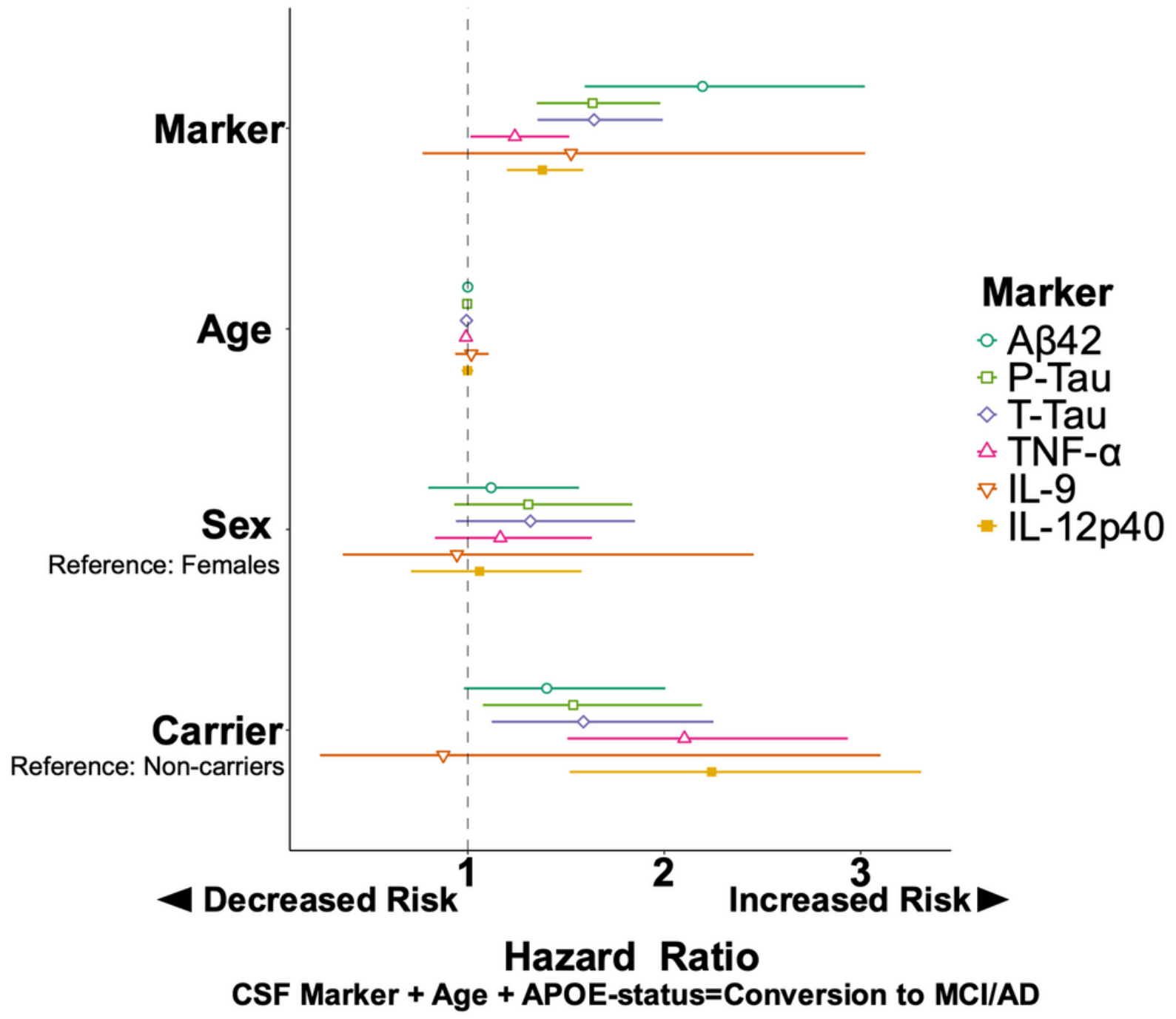

Figure 1

Forest plot highlighting predictive power of CSF inflammatory markers as predictors of conversion. Each model tested is represented by a line in the plot where color and shape differentiate our variables of interest. Line of no effect $(H R=1.0)$ is represented by dotted line. Variable hazard ratios (per IQR) visualize how the ratio magnitude of inflammatory markers TNF- $\mathrm{V}$, IL-9, and IL-12p40 compare to the magnitudes of the ratios of $A D$ biomarkers. 


\section{TNF- $\alpha^{\star}$ Sex}

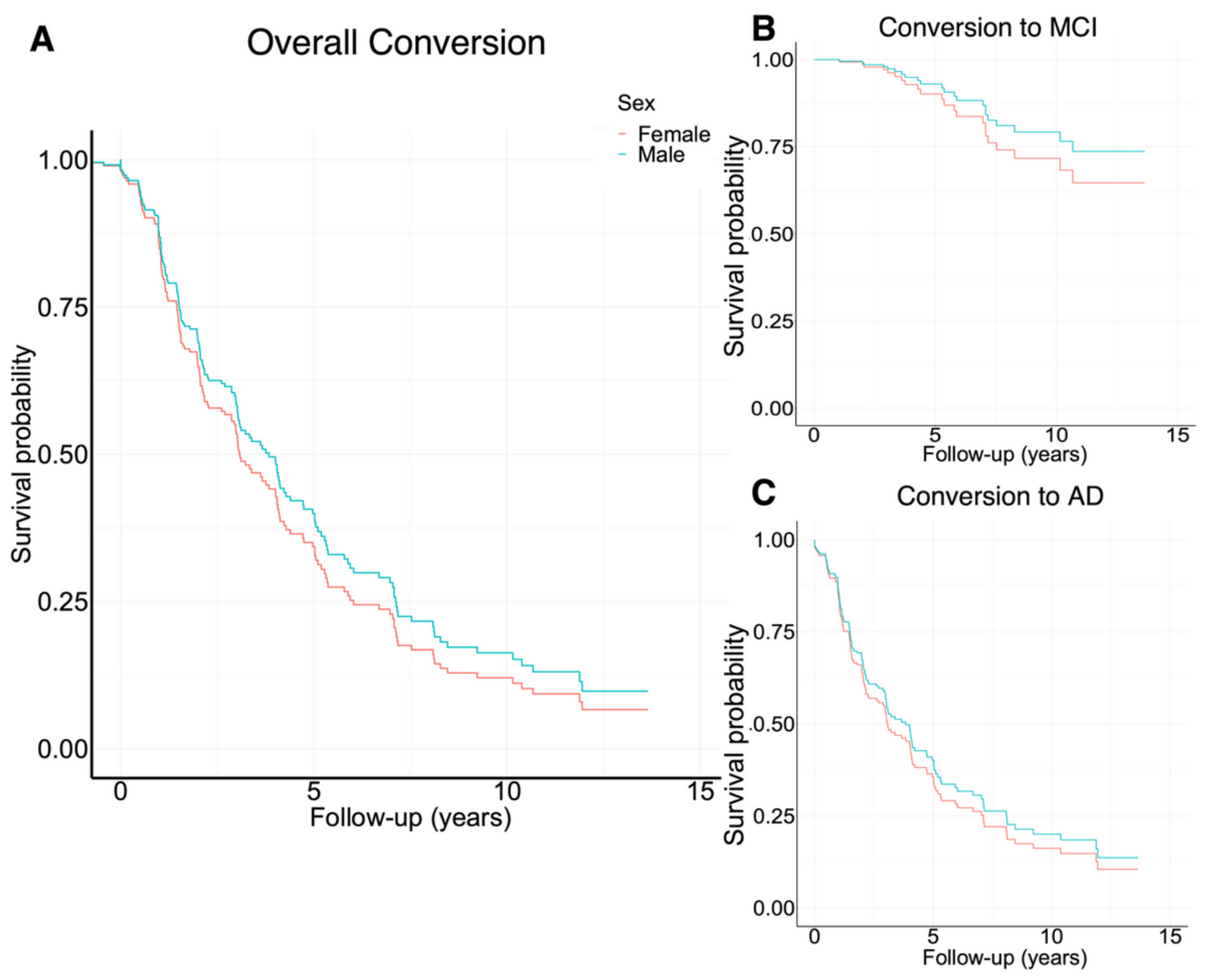

Figure 2

Survival curves for CSF inflammatory marker, TNF-a. Panel A shows survival probabilities split between sex. Red represents females and blue, males, to better show the interaction effect between sexes of overall conversion to either $\mathrm{MCl}$ or $\mathrm{AD}$. Females with high levels of TNF-a tended to have later conversion times. Results were split by conversion to either $\mathrm{MCl}$ (panel $\mathrm{B}$ ) or $\mathrm{AD}$ (panel $\mathrm{A}$ ) with conversion to $\mathrm{MCl}$ not significant. 


\section{IL-9*APOE4 Status}

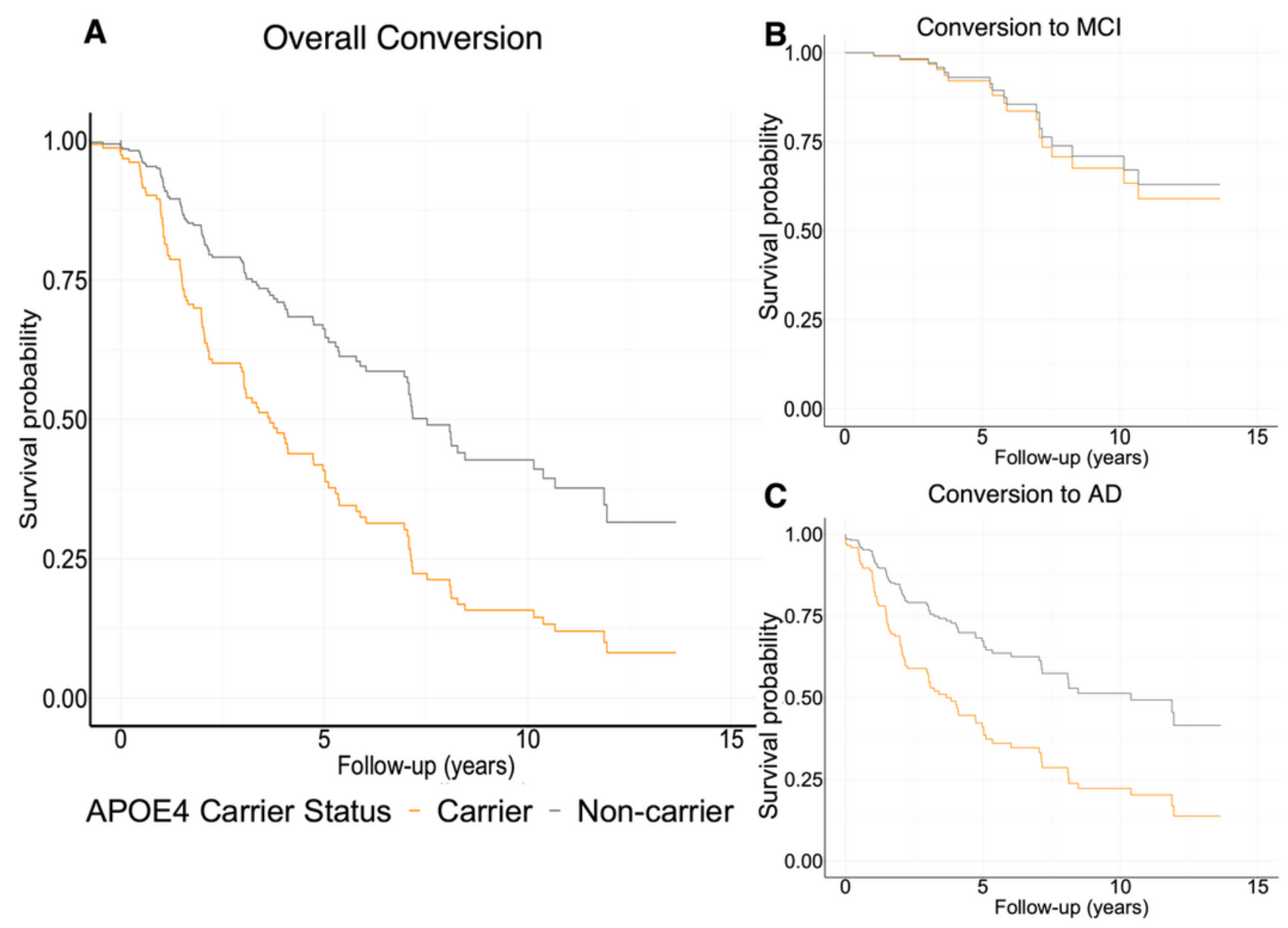

\section{Figure 3}

Survival curves for CSF inflammatory marker, IL-9. Panel A displays overall conversion to either $\mathrm{MCl}$ or $A D$, split between APOE4 carriers and noncarriers. Orange represents APOE4 carriers, and grey, noncarriers, illustrate that APOE4 carriers convert sooner in both the high and lower groups. When split between conversion status, these results were driven by conversion to $\mathrm{MCl}$ (Panel $\mathrm{B}$ ). Conversion to $\mathrm{AD}$ was not significant (Panel C). 


\section{IL-12p40*APOE4 Status}

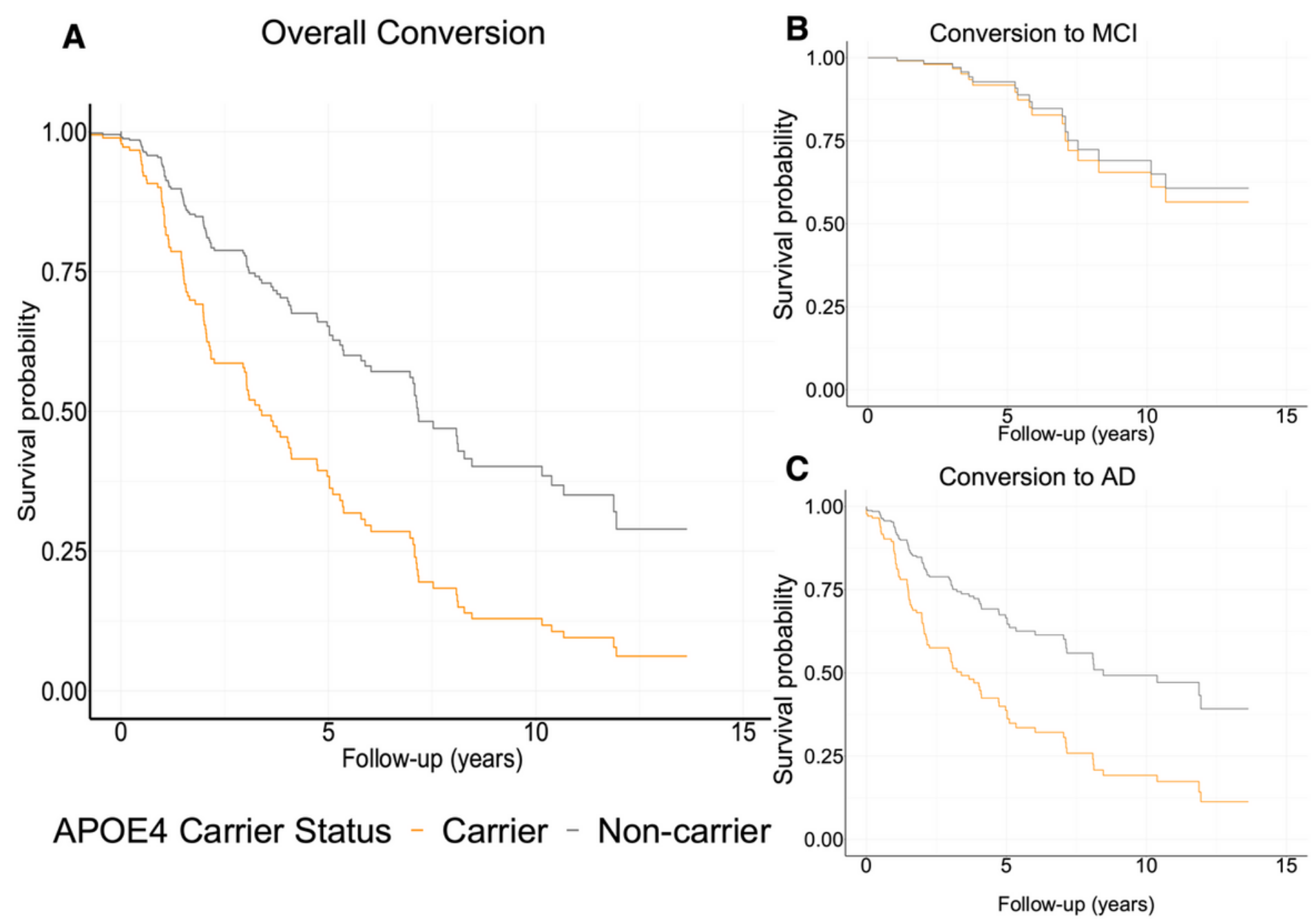

Figure 4

Survival curves for CSF inflammatory marker, IL-12p40. Panel A displays conversion to either $\mathrm{MCl}$ or $\mathrm{AD}$, split between APOE4 carriers and noncarriers. Orange represents APOE4 carriers, and grey represents noncarriers. When split between conversion status, these results were driven by conversion to AD (Panel C). Conversion to $\mathrm{MCl}$ was not significant (Panel B). 


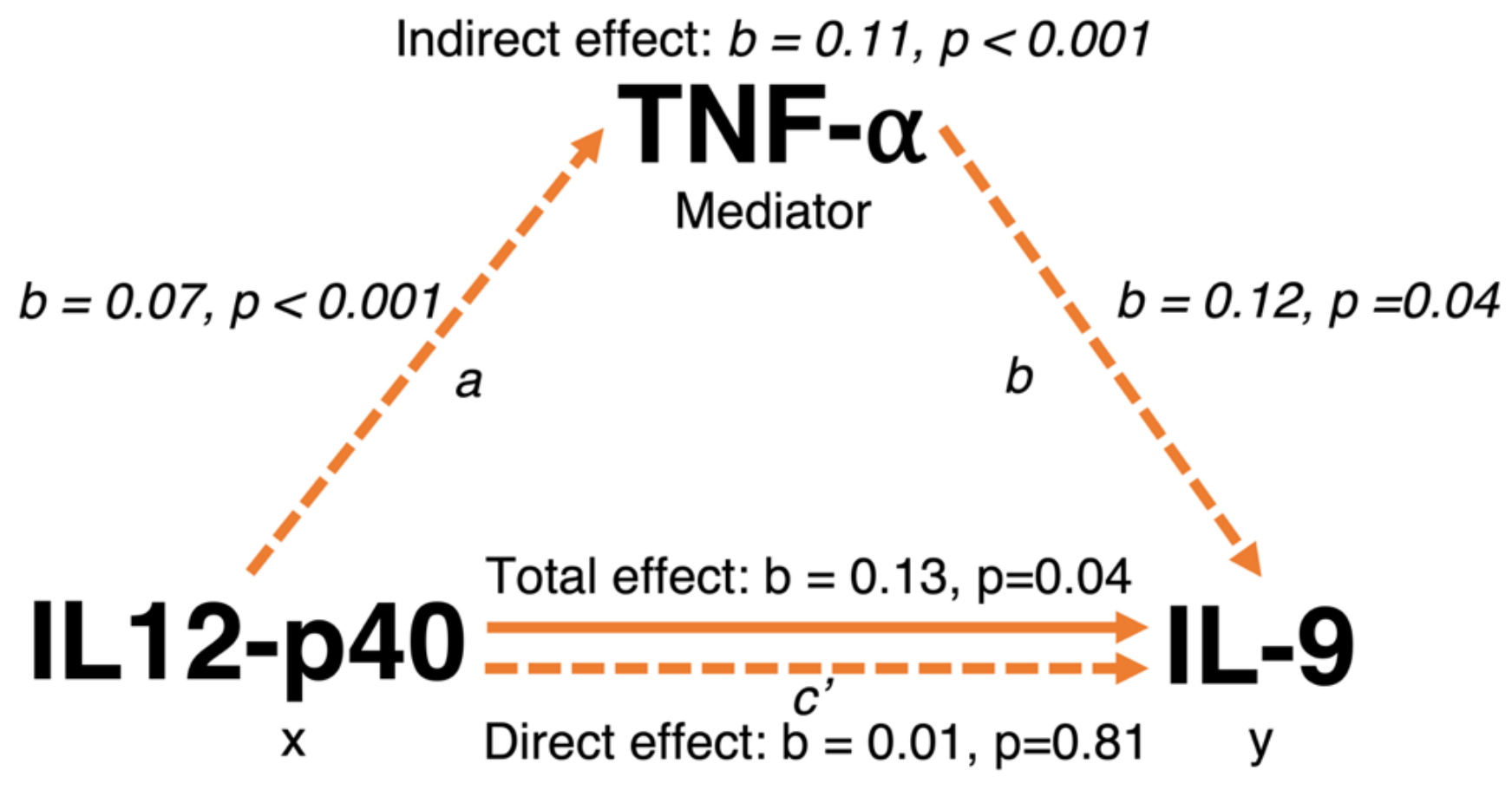

Figure 5

TNF- $\mathrm{a}$ mediates relationship between IL12-p40 and IL-9. The figure depicts the relationship between the independent, mediator, and dependent variables. The mediation analysis partitions the total variance (total effect) explained by the predictor into a part that is independent of the mediating variable (direct effect) and a part that is accounted for via the mediating variable (indirect effect). a represents the "a" path and $b$ represents the " $b$ " path. A bootstrapped mediation analysis revealed that TNF-a significantly mediated the relationship between inflammatory cytokines IL12-p40 (independent) and IL-9 (dependent) variables. 